\title{
Krasnosel'skii-type fixed point theorems with applications to Volterra integral equations
}

\author{
Nawab Hussain ${ }^{* *}$ and Mohamed Aziz Taoudi ${ }^{2,3}$ \\ Dedicated to Professor Wataru Takahashi on the occasion of his seventieth birthday
}

"Correspondence:

nhusain@kau.edu.sa

${ }^{1}$ Department of Mathematics, King

Abdulaziz University, P.O. Box 80203

Jeddah, 21589, Saudi Arabia

Full list of author information is

available at the end of the article

\begin{abstract}
In this paper we present some fixed point results for the sum $S+T$ of two mappings where $S$ is a strict contraction and $T$ is not necessarily weakly compact and satisfies a new condition formulated in terms of an axiomatic measure of weak

noncompactness. Our fixed point results extend and improve several earlier results in the literature. In particular, our results encompass the analogues of Krasnosel'skii's and Sadovskii's fixed point theorems for sequentially weakly continuous mappings and a number of their generalizations. Finally, an application to integral equations is given to illustrate the usability of the obtained results.
\end{abstract}

MSC: 37C25; 40D05; 31B10

Keywords: weakly noncompact; weakly continuous; $w$-contractive; condensing mapping; fixed point

\section{Introduction}

In 1930, Schauder proved that every continuous and compact mapping from a nonempty closed convex subset of a Banach space to itself has a fixed point. This result remained an important tool to solve differential and integral equations. In several situations, where the condition of compactness creates some difficulties, the Tychonov fixed point theorem appears as a good alternative. It asserts that every weakly continuous and weakly compact mapping from a nonempty closed convex subset of a Banach space to itself has a fixed point. As long as the Banach space is reflexive, the weak compactness offers no problem since every bounded subset is relatively weakly compact and therefore the weak continuity suffices to prove nice existence results for differential and integral equations $[1,2]$. In 1977, De Blasi [3] introduced the concept of measure of weak noncompactness and proved the analogue of Sadovskii's fixed point theorem for the weak topology (see also [4]). As stressed in [5], in many applications, it is always not possible to show the weak continuity of the involved mappings, while the sequential weak continuity offers no problem. This is mainly due to the fact that Lebesgue's dominated convergence theorem is valid for sequences but not for nets. Recall that a mapping between two Banach spaces is sequentially weakly continuous if it maps weakly convergent sequences into weakly convergent sequences. So, Arino, Gautier and Penot proved the analogue of Schauder's fixed point theorem for sequentially weakly continuous mappings. Since then, several fixed point theorems have been proved for sequentially weakly continuous mappings of Darbo type, Sadovskii type [6, 7] and Krasnosel'skii type [8-12] and many 
others. More recently, a generalized version of Sadovskii's fixed point theorem for sequentially weakly continuous mappings has been proved in [13]. Based on the concept of power-convex condensing mapping, this new fixed point theorem allowed, in many applications, to avoid some contractiveness conditions generated by the use of classical Sadovskii's fixed point theorem. Roughly speaking, the idea was to reason on the iterates of the given mapping instead of the mapping itself. Stimulated by some real world applications, we introduce the concept of power-convex condensing pair of sequentially weakly continuous mappings. We prove some fixed point theorems for the sum $S+T$ where $S$ is a strict contraction while $T$ is power-convex condensing w.r.t. $S$. In some applications, the concepts of ws-compactness and ww-compactness seem to be more practical than the sequential weak continuity. These concepts arise naturally in the study of both integral and partial differential equations in nonreflexive Banach spaces (see $[7,14-16]$ and the references therein). We prove some fixed point theorems for the sum $S+T$ where $S$ is a ww-compact strict contraction while $T$ is ws-compact and power-convex condensing w.r.t. $S$ without the sequential weak continuity of the involved mappings. The presented results extend all the fixed point theorems quoted above. As an application, we investigate the existence of continuous solutions to a perturbed Volterra integral equation which extends the corresponding results of [13] and many others. For convenience, we first recall some basic concepts and notations. Let $E$ be a Banach space, let $\Omega_{E}$ be the collection of all nonempty bounded subsets of $E$, and let $\mathcal{W}_{E}$ be the subset of $\Omega_{E}$ consisting of all weakly compact subsets of $E$. Let $B_{r}$ denote the closed ball in $E$ centered at 0 with radius $r>0$. The De Blasi [3] measure of weak noncompactness is the map $w: \Omega_{E} \rightarrow[0, \infty)$ defined by

$$
w(A)=\inf \left\{r>0: \text { there exists a set } W \in \mathcal{W}_{E} \text { such that } A \subseteq W+B_{r}\right\}
$$

for all $A \in \Omega_{E}$. For completeness, we enumerate some properties of $w$ which we will tacitly use in the sequel. Let $A, B \in \Omega_{E}$, then we have the following:

(1) $w(A)=0$ if and only if $A$ is relatively weakly compact;

(2) $w(A)=w\left(\bar{A}^{w}\right)$, where $\bar{A}^{w}$ is the weak closure of $A$;

(3) $w(\operatorname{co}(A))=w(A)$, where $\operatorname{co}(A)$ denotes the convex hull of $A$;

(4) if $A \subset B$, then $w(A) \leq w(B)$;

(5) $w(A \cup B)=\max \{w(A), w(B)\}$;

(6) $w(\lambda A)=|\lambda| w(A)$ for $\lambda \in \mathbb{R}$, where $\lambda A=\{\lambda x: x \in A\}$;

(7) $w(A+B) \leq w(A)+w(B)$, where $A+B=\{x+y: x \in A, y \in B\}$;

(8) if $A_{n}$ is a sequence of nonempty, weakly closed subsets of $E$ with $A_{1}$ bounded and $A_{1} \supseteq A_{2} \supseteq \cdots \supseteq A_{n} \cdots$ with $\lim _{n \rightarrow \infty} w\left(A_{n}\right)=0$, then $\bigcap_{n=1}^{\infty} A_{n} \neq \emptyset$ and $w\left(\bigcap_{n=1}^{\infty} A_{n}\right)=0$.

By a measure of weak noncompactness, we mean a map $\mu: \Omega_{E} \rightarrow \mathbb{R}^{+}$satisfying the properties (1)-(8) quoted above. In what follows, we need the following definition. Let $E$ be a Banach space, let $M$ be a nonempty closed convex subset of $E$, and let $S, T: M \rightarrow E$ be two nonlinear mappings and $x_{0} \in E$. For any $N \subseteq M$, we set

$$
\mathcal{F}^{\left(1, x_{0}\right)}(T, S, N)=\{x \in M: x=S x+T y \text { for some } y \in N\}
$$


and

$$
\mathcal{F}^{\left(n, x_{0}\right)}(T, S, N)=\mathcal{F}^{\left(1, x_{0}\right)}\left(T, S, \overline{c o}\left(\mathcal{F}^{\left(n-1, x_{0}\right)}(T, S, N) \cup\left\{x_{0}\right\}\right)\right)
$$

for $n=2,3, \ldots$.

Definition 1.1 Let $E$ be a Banach space, let $M$ be a nonempty closed convex subset of $E$, and let $\mu$ be a measure of weak noncompactness on $E$. Let $T, S: M \rightarrow E$ be two bounded mappings (i.e., they take bounded sets into bounded ones) and $x_{0} \in M$. We say that $T$ is an $S$-convex-power condensing operator about $x_{0}$ and $n_{0}$ w.r.t. $\mu$ if for any bounded set $N \subseteq M$ with $\mu(N)>0$ we have

$$
\mu\left(\mathcal{F}^{\left(n_{0}, x_{0}\right)}(T, S, N)\right)<\mu(N)
$$

Obviously, $T: M \rightarrow M$ is power-convex condensing with respect to $\mu$ about $x_{0}$ and $n_{0}$ [13] if and only if it is a 0 -convex-power condensing operator about $x_{0}$ and $n_{0}$ w.r.t. $\mu$.

The following results are crucial for our purposes. We first state a theorem of Ambrosetti type (see [17] for a proof).

Theorem 1.1 Let $E$ be a Banach space and let $H \subseteq C([0, T], E)$ be bounded and equicontinuous. Then the map $t \rightarrow w(H(t))$ is continuous on $[0, T]$ and

$$
w(H)=\sup _{t \in[0, T]} w(H(t))=w(H[0, T])
$$

where $H(t)=\{h(t): h \in H\}$ and $H[0, T]=\bigcup_{t \in[0, T]}\{h(t): h \in H\}$.

Theorem 1.2 [18, Theorem 9] Let $S$ be a Hausdorff compact space and E be a Banach space. A bounded sequence $\left(f_{n}\right) \subset C(S, E)$ converges weakly to $f \in C(S, E)$ if and only if, for every $t \in S$, the sequence $\left(f_{n}(t)\right)$ converges weakly (in $\left.E\right)$ to $f(t)$.

Definition 1.2 A mapping $T: D(T) \subset X \rightarrow X$ is called $k$-Lipschitzian if $\|T x-T y\| \leq k \| x-$ $y \|$ for all $x, y \in D(T) . T$ is called strict contraction if $k \in[0,1)$ and nonexpansive if $k=1$.

Lemma 1.1 [11] Let $M$ be a subset of $E$ and let $T: M \rightarrow$ E be a $k$-Lipschitzian map. Assume that $T$ is a sequentially weakly continuous map. Then $w(T(A)) \leq k w(A)$ for each bounded subset A of $M$; here, $w(\cdot)$ stands for the De Blasi measure of weak noncompactness.

Definition 1.3 We say that $T: D(T) \subset X \rightarrow X$ is demiclosed if for any sequence $\left\{x_{n}\right\}$ weakly convergent to an element $x^{*} \in D(T)$ with $\left\{T x_{n}\right\}$ norm-convergent to an element $y$, then $T x^{*}=y$.

Theorem 1.3 [19, Theorem 5.1.2] Let $M$ be a bounded closed convex subset of a Banach space $X$, and let $T$ be a nonexpansive mapping of $M$ into $M$. Then, for each $\varepsilon>0$, there is an $x_{\varepsilon} \in M$ such that $\left\|T x_{\varepsilon}-x_{\varepsilon}\right\|<\varepsilon$. 


\section{Fixed point theory for the sum in the weak topology}

Theorem 2.1 Let $M$ be a nonempty bounded closed convex subset of a Banach space $E$, and let $\mu$ be a measure of weak noncompactness on E. Suppose that $T: M \rightarrow E$ and $S: E \rightarrow E$ are two mappings satisfying:

(i) $T$ is sequentially weakly continuous,

(ii) $S$ is a strict contraction,

(iii) there are an integer $n_{0}$ and a vector $x_{0} \in E$ such that $T$ is $S$-power-convex condensing w.r.t. $\mu$ about $x_{0}$ and $n_{0}$,

(iv) if $x=S x+T y$, for some $y \in M$, then $x \in M$,

(v) if $\left\{x_{n}\right\}$ is a sequence in $\mathcal{F}^{\left(n_{0}, x_{0}\right)}(T, S, M)$ such that $x_{n} \rightarrow x$, then $S x_{n} \rightarrow S x$.

Then $T+S$ has at least one fixed point in $M$.

Proof Let $y \in M$. The map which assigns to each $x \in E$ the value $S x+T y$ defines a strict contraction mapping from $E$ into itself and so it has a unique fixed point in $E$ by the contraction mapping principle [19]. Let us denote by $\tau: M \rightarrow E$ the map which assigns to each $y \in M$ the unique point in $M$ such that $\tau(y)=S \tau(y)+T y$. From assumption (iv) we infer that $\tau(M) \subset M$. Notice

$$
\tau(N)=\mathcal{F}^{\left(1, x_{0}\right)}(T, S, N)
$$

for all $N \subset M$. Let

$$
\Gamma=\left\{N \subseteq M, \overline{c o}(N)=N, x_{0} \in N \text { and } \tau(N) \subseteq N\right\}
$$

The set $\Gamma$ is nonempty since $M \in \Gamma$. Set $C=\bigcap_{N \in \Gamma} N$. Now we show that for any positive integer $n$ we have

$$
C=\overline{c o}\left(\mathcal{F}^{\left(n, x_{0}\right)}(T, S, C) \cup\left\{x_{0}\right\}\right)
$$

To see this, we proceed by induction. Clearly $C$ is a closed convex subset of $M$ and $\tau(C) \subseteq C$. Thus $C \in \Gamma$. This implies $\overline{c o}\left(\tau(C) \cup\left\{x_{0}\right\}\right) \subseteq C$. Hence $\tau\left(\overline{c o}\left(\tau(C) \cup\left\{x_{0}\right\}\right)\right) \subseteq$ $\tau(C) \subseteq \overline{c o}\left(\tau(C) \cup\left\{x_{0}\right\}\right)$. Consequently, $\overline{c o}\left(\tau(C) \cup\left\{x_{0}\right\}\right) \in \Gamma$. Hence $C \subseteq \overline{c o}\left(\tau(C) \cup\left\{x_{0}\right\}\right)$. As a result $\overline{c o}\left(\tau(C) \cup\left\{x_{0}\right\}\right)=C$. This shows that $\mathcal{P}(1)$ holds. Let $n$ be fixed and suppose $\mathcal{P}(n)$ holds. This implies

$$
\begin{aligned}
\mathcal{F}^{\left(n+1, x_{0}\right)}(T, S, C) & =\mathcal{F}^{\left(1, x_{0}\right)}\left(T, S, \overline{c o}\left(\mathcal{F}^{\left(n, x_{0}\right)}(T, S, C) \cup\left\{x_{0}\right\}\right)\right) \\
& =\tau\left(\overline{c o}\left(\mathcal{F}^{\left(n, x_{0}\right)}(T, S, C) \cup\left\{x_{0}\right\}\right)\right) \\
& =\tau(C) .
\end{aligned}
$$

Consequently,

$$
\overline{c o}\left(\mathcal{F}^{\left(n+1, x_{0}\right)}(T, S, C) \cup\left\{x_{0}\right\}\right)=\overline{c o}\left(\tau(C) \cup\left\{x_{0}\right\}\right)=C .
$$

Thus, for all $n \geq 1$ we have

$$
C=\overline{c o}\left(\mathcal{F}^{\left(n, x_{0}\right)}(T, S, C) \cup\left\{x_{0}\right\}\right) .
$$


Using the properties of the measure of weak noncompactness, we get

$$
\mu(C)=\mu\left(\overline{c o}\left(\mathcal{F}^{\left(n_{0}, x_{0}\right)}(T, S, C) \cup\left\{x_{0}\right\}\right)\right),
$$

which yields that $C$ is weakly compact. We claim now that $\tau: C \rightarrow C$ is sequentially weakly continuous. Indeed, let $\left\{y_{n}\right\}$ be a sequence in $C$ such that $y_{n} \rightarrow y$ in $C$. Since $\tau(C) \subseteq C$, then there exists a subsequence $\left\{y_{n_{k}}\right\}$ of $\left\{y_{n}\right\}$ such that $\tau y_{n_{k}} \rightarrow z$ for some $z \in C$. By (v) $S \tau y_{n_{k}} \rightarrow$ $S z$. Also from (i) it follows that $T y_{n_{k}} \rightarrow T y$ and hence the equality $\tau y_{n_{k}}=S \tau\left(y_{n_{k}}\right)+T y_{n_{k}}$ gives us $z=S z+T y$. By uniqueness, we conclude that $\tau(y)=z$.

Accordingly, we have

$$
\tau\left(y_{n_{k}}\right) \rightarrow \tau(y)
$$

Now a standard argument shows that

$$
\tau\left(y_{n}\right) \rightarrow \tau(y) .
$$

Suppose the contrary, then there exist a weak neighborhood $N^{w}$ of $\tau(y)$ and a subsequence $\left\{y_{n_{j}}\right\}$ of $\left\{y_{n}\right\}$ such that $\tau\left(y_{n_{j}}\right) \notin N^{w}$ for all $j \geq 1$. Naturally, $\left(y_{n_{j}}\right)$ converges weakly to $y$. Then, arguing as before, we may extract a subsequence $\left(y_{n_{j_{k}}}\right)$ of $\left(y_{n_{j}}\right)$ such that $\tau\left(y_{n_{j_{k}}}\right) \rightarrow \tau(y)$, which is absurd since $\tau\left(y_{n_{j_{k}}}\right) \notin N^{w}$ for all $k \geq 1$. Finally, $\tau$ is weakly sequentially continuous. Applying the Arino-Gautier-Penot fixed point theorem [5], we infer that there exists $x \in C$ such that

$$
x=\tau(x)=S(\tau(x))+T x=S x+T x .
$$

It is worthwhile to emphasize that Theorem 2.1 encompasses a lot of previously known results. In particular, if we take $B=0$ in Theorem 2.1, we recapture the following fixed point theorem, which was proved in [13, Theorem 2.1].

Corollary 2.1 Let $M$ be a nonempty bounded closed convex subset of a Banach space $X$. Suppose that $T: M \rightarrow M$ is weakly sequentially continuous and there exist an integer $n_{0}$ and a vector $x_{0} \in E$ such that $T$ is power-convex condensing about $x_{0}$ and $n_{0}$. Then $T$ has at least one fixed point in $M$.

Another consequence of Theorem 2.1 is the following result, which is a sharpening of [20, Theorem 2.9].

Corollary 2.2 Let $M$ be a nonempty bounded closed convex subset of a Banach space E. Suppose that $T: M \rightarrow E$ and $S: E \rightarrow E$ are two mappings satisfying:

(i) $T$ is sequentially weakly continuous,

(ii) $S$ is a strict contraction with constant $k$,

(iii) there exists an integer $n_{0}$ such that $\mathcal{F}^{\left(n_{0}, x_{0}\right)}(T, S, M)$ is relatively weakly compact,

(iv) if $x=S x+T y$, for some $y \in M$, then $x \in M$,

(v) if $\left\{x_{n}\right\}$ is a sequence in $\mathcal{F}^{\left(n_{0}, x_{0}\right)}(T, S, M)$ such that $x_{n} \rightarrow x$, then $S x_{n} \rightarrow S x$.

Then $T+S$ has at least one fixed point in $M$. 
In order to state another consequence of Theorem 2.1, the following abstract lemma is very useful.

Lemma 2.1 Assume that the conditions (i), (ii) and (iv) of Theorem 2.1 hold. If, moreover, $S$ is sequentially weakly continuous and $T(M)$ is relatively weakly compact, then the set $\mathcal{F}:=\mathcal{F}^{\left(1, x_{0}\right)}(T, S, M):=\{x \in M: x=S x+$ Ty for some $y \in M\}$ is relatively weakly compact.

Proof From the definition of $\mathcal{F}$ it follows that

$$
\mathcal{F} \subset S(\mathcal{F})+T(M)
$$

Keeping in mind that $T(M)$ is relatively weakly compact and using Lemma 1.1, we get

$$
\begin{aligned}
w(\mathcal{F}) & \leq w(S(\mathcal{F}))+w(T(M)) \\
& =w(S(\mathcal{F})) \\
& \leq k w(\mathcal{F}) .
\end{aligned}
$$

Since $0 \leq k<1$, then $w(\mathcal{F})=0$ and therefore $\mathcal{F}$ is relatively compact.

On the basis of Lemma 2.1, the following Krasnosel'skii-type fixed point theorem follows from Theorem 2.1.

Corollary 2.3 [8, Theorem 2.1] Let $M$ be a nonempty bounded closed convex subset of a Banach space E. Suppose that $T: M \rightarrow E$ and $S: E \rightarrow E$ are two sequentially weakly continuous mappings satisfying:

(i) $T(M)$ is relatively weakly compact,

(ii) $S$ is a strict contraction,

(iii) if $x=S x+T y$, for some $y \in M$, then $x \in M$.

Then $T+S$ has at least one fixed point in $M$.

Now we consider the case where $S$ is nonexpansive.

Theorem 2.2 Let $E$ be a Banach space and $\mu$ be a measure of weak noncompactness on $E$. Let $M$ be a nonempty bounded closed convex subset of $E$, and let $T, S: M \rightarrow X$ be two sequentially weakly continuous mappings satisfying:

(i) there are an integer $n$ and a vector $x_{0} \in E$ such that $T$ is S-power-convex condensing w.r.t. $\mu$,

(ii) $S$ is a nonexpansive mapping,

(iii) if $\left(x_{n}\right)$ is a sequence of $M$ such that $\left((I-S) x_{n}\right)$ is weakly convergent, then the sequence $\left(x_{n}\right)$ has a weakly convergent subsequence,

(iv) $I-S$ is injective and demiclosed,

(v) $T x+$ Sy $\in M$ for all $x, y \in M$.

Then $T+S$ has at least one fixed point in $M$.

Proof Let $z \in T(M)$. The map which assigns to each $x \in M$ the value $S x+z$ defines a nonexpansive mapping from $M$ into $M$. In view of Theorem 1.3, there exists a sequence $\left(x_{n}\right)$ 
in $M$ such that

$$
(I-S) x_{n}-z \rightarrow 0
$$

By assumption (iii) we have that $\left(x_{n}\right)$ has a subsequence, say $\left(x_{n_{k}}\right)$, which converges to some $x \in M$. Since $(I-S)$ is demiclosed, then $z=(I-S) x$. Hence $z \in(I-S) M$. Consequently, $T(M) \subseteq(I-S)(M)$. Let us denote by $\tau$ the map which assigns to each $y \in M$ the point $\tau(y) \in M$ such that $(I-S) \tau(y)=T y$. Since $I-S$ is injective, then $\tau: M \rightarrow M$ is well defined. It is easily seen that

$$
\tau(N)=\mathcal{F}^{\left(1, x_{0}\right)}(T, S, N)
$$

for all $N \subset M$. Let

$$
\Gamma=\left\{N \subseteq M, \overline{c o}(N)=N, x_{0} \in N \text { and } \tau(N) \subseteq N\right\}
$$

The set $\Gamma$ is nonempty since $M \in \Gamma$. Set $C=\bigcap_{N \in \Gamma} N$. Proceeding by induction, we have

$$
C=\overline{c o}\left(\mathcal{F}^{\left(n, x_{0}\right)}(T, S, C) \cup\left\{x_{0}\right\}\right)
$$

for any integer $n$. Using the properties of the measure of weak noncompactness, we get

$$
\mu(C)=\mu\left(\overline{c o}\left(\mathcal{F}^{\left(n, x_{0}\right)}(T, S, C) \cup\left\{x_{0}\right\}\right)\right),
$$

which yields that $C$ is weakly compact. The reasoning in Theorem 2.1 shows that $\tau: C \rightarrow C$ is sequentially weakly continuous. Applying the Arino-Gautier-Penot fixed point theorem, we infer that there exists $x \in C$ such that

$$
x=\tau(x)=S(\tau(x))+T x=S x+T x
$$

An easy consequence of Theorem 2.2 is the following.

Corollary 2.4 Let $M$ be a nonempty bounded closed convex subset of a reflexive Banach space $E$, and let $\mu$ be a measure of weak noncompactness on $E$. Suppose that $T, S: M \rightarrow E$ are two continuous mappings satisfying:

(i) there are an integer $n$ and a vector $x_{0} \in E$ such that $T$ is $S$-power-convex condensing w.r.t. $\mu$,

(ii) $S$ is nonexpansive,

(iii) $I-S$ is injective and demiclosed,

(iv) $T x+S y \in M$ for all $x, y \in M$.

Then $S+T$ has at least one fixed point in $M$.

Proof Keeping in mind that every bounded subset in a reflexive Banach space is relatively weakly compact, the result follows from Theorem 2.2 .

Corollary 2.5 Let $M$ be a nonempty bounded closed convex subset of a uniformly convex Banach space E. Suppose that $T, S: M \rightarrow$ E are two continuous mappings satisfying: 
(i) there are an integer $n$ and a vector $x_{0} \in E$ such that $T$ is $S$-power-convex condensing w.r.t. $\mu$,

(ii) $S$ is nonexpansive and $I-S$ is injective,

(iii) $T x+S y \in M$ for all $x, y \in M$.

Then $T+S$ has at least one fixed point in $M$.

Proof Note that in a uniformly convex space we have $S$ is nonexpansive implies $I-S$ is demiclosed (see [21]). Moreover, every uniformly convex Banach space is reflexive. The result follows from Corollary 2.4.

In some applications, the sequential weak continuity condition is not easy to be verified. We thus consider the following two conditions: let $T: D(T) \subset E \rightarrow E$ be a map.

(H1) If $\left(x_{n}\right)_{n \in \mathbb{N}}$ is a weakly convergent sequence in $D(T)$, then $\left(T x_{n}\right)_{n \in \mathbb{N}}$ has a strongly convergent subsequence in $E$.

(H2) If $\left(x_{n}\right)_{n \in \mathbb{N}}$ is a weakly convergent sequence in $D(T)$, then $\left(T x_{n}\right)_{n \in \mathbb{N}}$ has a weakly convergent subsequence in $E$.

Remark 2.1 Continuous mappings satisfying (H1) are called ws-compact mappings and continuous mappings satisfying $(\mathrm{H} 2)$ are called ww-compact mappings. Note that wscompact and ww-compact mappings are not necessarily weakly continuous [12].

Now we state the following result.

Theorem 2.3 Let $M$ be a nonempty bounded closed convex subset of a Banach space $E$, and let $\mu$ be a measure of weak noncompactness on E. Suppose that $T: M \rightarrow E$ and $S: E \rightarrow E$ are two continuous mappings satisfying:

(i) $T$ verifies ( $\mathrm{H} 1)$,

(ii) $S$ is a strict contraction verifying $(\mathrm{H} 2)$,

(iii) there are an integer $n_{0}$ and a vector $x_{0} \in E$ such that $T$ is $S$-power-convex condensing w.r.t. $\mu$ about $x_{0}$ and $n_{0}$,

(iv) if $x=S x+T y$, for some $y \in M$, then $x \in M$.

Then $T+S$ has at least one fixed point in $M$.

Proof Let $y \in M$. The map which assigns to each $x \in E$ the value $S x+T y$ defines a strict contraction mapping from $E$ into itself and so it has a unique fixed point in $E$ by the contraction mapping principle [19]. Let us denote by $\tau: M \rightarrow E$ the map which assigns to each $y \in M$ the unique point in $M$ such that $\tau(y)=S \tau(y)+T y$. From assumption (iv) we infer that $\tau(M) \subset M$. Notice

$$
\tau(N)=\mathcal{F}^{\left(1, x_{0}\right)}(T, S, N)
$$

for all $N \subset M$. Let

$$
\Gamma=\left\{N \subseteq M, \overline{c o}(N)=N, x_{0} \in N \text { and } \tau(N) \subseteq N\right\}
$$


The set $\Gamma$ is nonempty since $M \in \Gamma$. Set $C=\bigcap_{N \in \Gamma} N$. The reasoning in Theorem 2.1 shows that for any positive integer $n$ we have

$$
C=\overline{c o}\left(\mathcal{F}^{\left(n, x_{0}\right)}(T, S, C) \cup\left\{x_{0}\right\}\right) .
$$

Using the properties of the measure of weak noncompactness, we get

$$
\mu(C)=\mu\left(\overline{c o}\left(\mathcal{F}^{\left(n_{0}, x_{0}\right)}(T, S, C) \cup\left\{x_{0}\right\}\right)\right),
$$

which yields that $C$ is weakly compact. Put $D=\overline{c o}(\tau(C))$ the closed convex hull of $\tau(C)$. We claim that $D$ is compact. Indeed, let $\left\{x_{n}\right\}$ be a sequence in $C$. Since $C$ is weakly compact, then, up to a subsequence, we may assume that $\left\{x_{n}\right\}$ converges weakly to some $x \in C$. Bearing in mind that $T$ satisfies (H1), we infer that $\left\{T x_{n}\right\}$ has a norm convergent sequence, say $\left\{T x_{n_{k}}\right\}$, which converges to some $z \in E$. From the equality

$$
\tau\left(x_{n_{k}}\right)=S \tau\left(x_{n_{k}}\right)+T x_{n_{k}},
$$

it follows that for any $p, q$ we have

$$
\begin{aligned}
\left\|\tau\left(x_{n_{p}}\right)-\tau\left(x_{n_{q}}\right)\right\| & =\left\|S \tau\left(x_{n_{p}}\right)-S \tau\left(x_{n_{q}}\right)+T x_{n_{p}}-T x_{n_{q}}\right\| \\
& \leq\left\|S \tau\left(x_{n_{p}}\right)-S \tau\left(x_{n_{q}}\right)\right\|+\left\|T x_{n_{p}}-T x_{n_{q}}\right\| \\
& \leq k\left\|\tau\left(x_{n_{p}}\right)-\tau\left(x_{n_{q}}\right)\right\|+\left\|T x_{n_{p}}-T x_{n_{q}}\right\| .
\end{aligned}
$$

Accordingly,

$$
\left\|\tau\left(x_{n_{p}}\right)-\tau\left(x_{n_{q}}\right)\right\| \leq \frac{1}{1-k}\left\|T x_{n_{p}}-T x_{n_{q}}\right\| .
$$

This implies that $\left\{\tau\left(x_{n_{k}}\right)\right\}$ is a Cauchy sequence in the Banach space $E$ and hence it is convergent. Thus, $\tau(C)$ is relatively compact and therefore $D=\overline{c o}(\tau(C))$ is compact (see [22]). We now claim that $\tau: D \rightarrow D$ is continuous. Indeed, let $\left\{x_{n}\right\}$ be a sequence in $D$ such that $x_{n} \rightarrow x$ in $D$. The continuity of $T$ guarantees that $T x_{n} \rightarrow T x$. Hence,

$$
\begin{aligned}
\left\|\tau\left(x_{n}\right)-\tau(x)\right\| & =\left\|S \tau\left(x_{n}\right)-S \tau(x)+T x_{n}-T x\right\| \\
& \leq\left\|S \tau\left(x_{n}\right)-S \tau(x)\right\|+\left\|T x_{n}-T x\right\| \\
& \leq k\left\|\tau\left(x_{n}\right)-\tau(x)\right\|+\left\|T x_{n}-T x\right\| .
\end{aligned}
$$

Accordingly,

$$
\left\|\tau\left(x_{n}\right)-\tau(x)\right\| \leq \frac{1}{1-k}\left\|T x_{n}-T x\right\| .
$$

Consequently, $\tau\left(x_{n}\right) \rightarrow \tau(x)$. This proves that $\tau: D \rightarrow D$ is continuous. The Schauder fixed point theorem guarantees the existence of $x \in C$ such that

$$
x=\tau(x)=S(\tau(x))+T x=S x+T x .
$$


The case $B=0$ in Theorem 2.3 corresponds to the following result, which was proved in [12, Theorem 2.1].

Corollary 2.6 Let $E$ be a Banach space and $\mu$ be a measure of weak noncompactness on $E$. Let $M \subset E$ be a nonempty closed convex and bounded subset, $x_{0} \in E$, and let $n_{0}$ be a positive integer. Suppose that $T: M \rightarrow M$ is power-convex condensing w.r.t. $\mu$ about $x_{0}$ and $n_{0}$. If $T$ is ws-compact, then there exists $x \in M$ such that $T x=x$.

Another consequence of Theorem 2.3 is the following.

Corollary 2.7 Let $M$ be a nonempty bounded closed convex subset of a Banach space E, and let $\mu$ be a measure of weak noncompactness on E. Suppose that $T: M \rightarrow E$ and $S: E \rightarrow$ E are two continuous mappings satisfying:

(i) $T$ verifies (H1),

(ii) $S$ is a strict contraction verifying $(\mathrm{H} 2)$,

(iii) there are an integer $n_{0}$ and a vector $x_{0}$ such that $\mathcal{F}^{\left(n_{0}, x_{0}\right)}(T, S, N)$ is relatively weakly compact,

(iv) if $x=S x+T y$, for some $y \in M$, then $x \in M$.

Then $T+S$ has at least one fixed point in $M$.

On the basis of Lemma 2.1 the following Krasnosel'skii-type fixed point theorem follows from Corollary 2.7.

Corollary 2.8 [15, Theorem 2.1] Let $M$ be a nonempty bounded closed convex subset of a Banach space E. Suppose that $T: M \rightarrow E$ and $S: E \rightarrow E$ are two continuous mappings satisfying:

(i) $T(M)$ is relatively weakly compact and $T$ satisfies $(\mathrm{H} 1)$,

(ii) $S$ is a strict contraction and $S$ verifies $(\mathrm{H} 2)$,

(iii) if $x=S x+T y$, for some $y \in M$, then $x \in M$.

Then $T+S$ has at least one fixed point in $M$.

\section{Application}

In this section we shall discuss the existence of weak solutions to the Volterra integral equation

$$
x(t)=f(x(t))+\int_{0}^{t} g(s, x(s)) d s, \quad t \in[0, T]
$$

here $g:[0, T] \times X \rightarrow X, f: X \rightarrow X$ and $x_{0} \in X$ with $X$ being a real Banach space. The integral in (3.1) is understood to be the Pettis integral and solutions to (3.1) will be sought in $E:=C([0, T], X)$.

This equation will be studied under the following assumptions:

(i) For each $t \in[0, T], g_{t}=g(t, \cdot)$ is sequentially weakly continuous (i.e., for each $t \in[0, T]$, for each weakly convergent sequence $\left(x_{n}\right)$, the sequence $g_{t}\left(x_{n}\right)$ is weakly convergent).

(ii) For each continuous $x:[0, T] \rightarrow E, g(\cdot, x(\cdot))$ is Pettis integrable on $[0, T]$. 
(iii) There exist $\alpha \in L^{1}[0, T]$ and $\theta:[0,+\infty) \rightarrow(0,+\infty)$ a nondecreasing continuous function such that $\|g(s, u)\| \leq \alpha(s) \theta(\|u\|)$ for a.e. $s \in[0, T]$, and all $u \in X$, with

$$
\int_{0}^{T} \alpha(s) d s<\int_{0}^{\infty} \frac{d x}{\theta(x)}
$$

(iv) There is a constant $\lambda \geq 0$ such that for any bounded subset $S$ of $X$ and for any $t \in[0, T]$, we have

$$
w(g([0, t] \times S)) \leq \lambda w(S)
$$

(v) $f: X \rightarrow X$ is sequentially weakly continuous.

(vi) There exists $k \in[0,1)$ such that $\|f(u)-f(v)\| \leq k\|u-v\|$ for all $u, v \in X$.

Theorem 3.1 Let X be a Banach space and suppose (i)-(vi) hold. Then (3.1) has a solution in $E=C([0, T], X)$.

Proof Let

$$
\begin{aligned}
M= & \{x \in C([0, T], X):\|x(t)\| \leq b(t) \text { for } t \in[0, T] \text { and } \\
& \|x(t)-x(s)\| \leq|b(t)-b(s)| \text { for } t, s \in[0, T]\},
\end{aligned}
$$

where

$$
b(t)=I^{-1}\left(\frac{1}{1-k} \int_{0}^{t} \alpha(s) d s\right) \text { and } I(z)=\int_{\frac{\|f(0)\|}{1-k}}^{z} \frac{d x}{\theta(x)} .
$$

Clearly,

$$
b^{\prime}(t)=\frac{1}{1-k} \alpha(t) \theta(b(t)) \quad \text { and } \quad b(0)=\frac{1}{1-k}\|f(0)\|
$$

for all $t \in[0, T]$. Also notice that $M$ is a closed, convex, bounded, equicontinuous subset of $C([0, T], X)$ with $0 \in C$. To allow the abstract formulation of equation (3.1), we define the following operators $S, T: C([0, T], X) \rightarrow C([0, T], X)$ by

$$
(T x)(t)=f(0)+\int_{0}^{t} g(s, x(s)) d s
$$

and

$$
(S x)(t)=f(x(t))-f(0) .
$$

Our strategy is to apply Theorem 2.1 to show the existence of a fixed point for the sum $S+T$ in $M$ which in turn is a continuous solution for equation (3.1). The proof will be divided into several steps.

Step 1: We show that $(x=S x+T y, y \in M)$ implies $x \in M$. 
Let $x \in C([0, T], X)$ such that $x=S x+T y$ with $y \in M$. For all $t \in[0, T]$ we have

$$
x(t)=f(x(t))+\int_{0}^{t} g(s, x(s)) d s .
$$

Hence,

$$
\begin{aligned}
\|x(t)\| & \leq\|f(x(t))\|+\int_{0}^{t}\|g(s, x(s))\| d s \\
& \leq\|f(0)\|+k\|x(t)\|+\int_{0}^{t} \alpha(s) \theta(\|y(s)\|) d s \\
& \leq\|f(0)\|+k\|x(t)\|+\int_{0}^{t} \alpha(s) \theta(b(s)) d s \\
& \leq\|f(0)\|+k\|x(t)\|+(1-k) \int_{0}^{t} b^{\prime}(s) d s \\
& \leq\|f(0)\|+k\|x(t)\|+(1-k)(b(t)-b(0)) \\
& \leq k\|x(t)\|+(1-k) b(t) .
\end{aligned}
$$

Accordingly,

$$
\|x(t)\| \leq b(t)
$$

On the other hand, let $t, s \in[0, T]$ with $t<s$ and let $x \in C([0, T], X)$. Then

$$
\begin{aligned}
\|x(t)-x(s)\| & \leq\|f(x(t))-f(x(s))\|+\int_{t}^{s}\|g(r, x(r))\| d r \\
& \leq k\|x(t)-x(s)\|+\int_{t}^{s} \alpha(r) \theta(\|x(r)\|) d r \\
& \leq k\|x(t)-x(s)\|+(1-k) \int_{t}^{s} b^{\prime}(r) d r \\
& \leq k\|x(t)-x(s)\|+(1-k)|b(s)-b(t)| .
\end{aligned}
$$

Accordingly,

$$
\|x(t)-x(s)\| \leq|b(t)-b(s)|
$$

Consequently, $x \in M$.

Step 2: Now we show that there is an integer $n_{0}$ such that $T$ is $S$-power-convex condensing w.r.t. $w$ about 0 and $n_{0}$, where $w$ is the De Blasi measure of weak noncompactness. To see this, notice, for each bounded set $\Omega \subseteq M$ and for each $t \in[0, T]$, that

$$
\begin{aligned}
\mathcal{F}^{(1,0)}(T, S, \Omega)(t)= & \left\{x(t), x \in \mathcal{F}^{(1,0)}(T, S, \Omega)\right\} \\
\subseteq & \left\{x(t)-f(x(t))+f(0), x \in \mathcal{F}^{(1,0)}(T, S, \Omega)\right\} \\
& +\left\{f(x(t))-f(0), x \in \mathcal{F}^{(1,0)}(T, S, \Omega)\right\} \\
\subseteq & T(\Omega)(t)+\left\{f(x(t))-f(0), x \in \mathcal{F}^{(1,0)}(T, S, \Omega)\right\} .
\end{aligned}
$$


Thus

$$
\alpha\left(\mathcal{F}^{(1,0)}(T, S, \Omega)(t)\right) \leq \alpha(T(\Omega)(t))+k \alpha\left(\mathcal{F}^{(1,0)}(T, S, \Omega)(t)\right) .
$$

Consequently,

$$
w\left(\mathcal{F}^{(1,0)}(T, S, \Omega)(t)\right) \leq \frac{1}{1-k} w(T(\Omega)(t)) .
$$

Further,

$$
\begin{aligned}
w(T(\Omega)(t)) & =w\left(\left\{f(0)+\int_{0}^{t} g(s, x(s)) d s: x \in \Omega\right\}\right) \\
& \leq w(t \overline{c o}\{g(s, x(s)): x \in \Omega, s \in[0, t]\}) \\
& =t w(\overline{c o}\{g(s, x(s)): x \in \Omega, s \in[0, t]\}) \\
& \leq t w(g([0, t] \times \Omega[0, t])) \\
& \leq t \lambda w(\Omega[0, t]) .
\end{aligned}
$$

Theorem 1.1 implies (since $M$ is equicontinuous) that

$$
w(T(\Omega)(t)) \leq t \lambda w(\Omega)
$$

Linking (3.7) and (3.8), we get

$$
w\left(\mathcal{F}^{(1,0)}(T, S, \Omega)(t)\right) \leq \frac{t \lambda}{1-k} w(\Omega) .
$$

Using (3.7) we obtain

$$
\begin{aligned}
w\left(\mathcal{F}^{(2,0)}(T, S, \Omega)(t)\right) & =w\left(\mathcal{F}^{(1,0)}\left(T, S, \overline{c o}\left(\mathcal{F}^{(1,0)}(T, S, \Omega) \cup\{0\}(t)\right)\right)\right) \\
& \leq \frac{1}{1-k} w\left(T\left(\overline{c o}\left(\mathcal{F}^{(1,0)}(T, S, \Omega) \cup\{0\}(t)\right)\right)\right) .
\end{aligned}
$$

Put $V=\overline{c o}\left(\mathcal{F}^{(1,0)}(T, S, \Omega) \cup\{0\}\right)$. The use of (3.8) yields

$$
\begin{aligned}
w\left(\mathcal{F}^{(2,0)}(T, S, \Omega)(t)\right) & \leq \frac{1}{1-k} w(T(V)(t)) \leq \frac{1}{1-k} w\left(\left\{f(0)+\int_{0}^{t} g(s, x(s)) d s: x \in V\right\}\right) \\
& \leq \frac{1}{1-k} w\left(\left\{\int_{0}^{t} g(s, x(s)) d s: x \in V\right\}\right) .
\end{aligned}
$$

Fix $t \in[0, T]$. We divide the interval $[0, t]$ into $m$ parts $0=t_{0}<t_{1}<\cdots<t_{m}=t$ in such a way that $\Delta t_{i}=t_{i}-t_{i-1}=\frac{t}{m}, i=1, \ldots, m$. For each $x \in V$, we have

$$
\begin{aligned}
\int_{0}^{t} g(s, x(s)) d s & =\sum_{i=1}^{m} \int_{t_{i-1}}^{t_{i}} g(s, x(s)) d s \in \sum_{i=1}^{m} \Delta t_{i} \overline{c o}\left\{g(s, x(s)): x \in V, s \in\left[t_{i-1}, t_{i}\right]\right\} \\
& \subseteq \sum_{i=1}^{m} \Delta t_{i} \overline{c o}\left(g\left(\left[t_{i-1}, t_{i}\right] \times V\left(\left[t_{i-1}, t_{i}\right]\right)\right)\right) .
\end{aligned}
$$


Using again Theorem 1.1, we infer that for each $i=2, \ldots, m$, there is an $s_{i} \in\left[t_{i-1}, t_{i}\right]$ such that

$$
\sup _{s \in\left[t_{i-1}, t_{i}\right]} w(V(s))=w\left(V\left[t_{i-1}, t_{i}\right]\right)=w\left(V\left(s_{i}\right)\right) .
$$

Consequently,

$$
\begin{aligned}
w\left(\left\{\int_{0}^{t} g(s, x(s)) d s: x \in V\right\}\right) & \leq \sum_{i=1}^{m} \Delta t_{i} w\left(\overline{c o}\left(g\left(\left[t_{i-1}, t_{i}\right] \times V\left(\left[t_{i-1}, t_{i}\right]\right)\right)\right)\right) \\
& \leq \lambda \sum_{i=1}^{m} \Delta t_{i} w\left(\overline{c o}\left(V\left(\left[t_{i-1}, t_{i}\right]\right)\right)\right) \\
& \leq \lambda \sum_{i=1}^{m} \Delta t_{i} w\left(V\left(s_{i}\right)\right) .
\end{aligned}
$$

On the other hand, if $m \rightarrow \infty$ then

$$
\sum_{i=1}^{m} \Delta t_{i} w\left(V\left(s_{i}\right)\right) \rightarrow \int_{0}^{t} w(V(s)) d s
$$

Thus,

$$
w\left(\left\{\int_{0}^{t} g(s, x(s)) d s: x \in V\right\}\right) \leq \int_{0}^{t} w(V(s)) d s .
$$

Using the regularity, the set additivity, the convex closure invariance of the De Blasi measure of weak noncompactness together with (3.8), we obtain

$$
w(V(s))=w\left(\mathcal{F}^{(1,0)}(T, S, \Omega)(s)\right) \leq \frac{s \lambda}{1-k} w(\Omega)
$$

and therefore

$$
\int_{0}^{t} w(V(s)) d s \leq \frac{\lambda}{1-k} \frac{t^{2}}{2} w(\Omega) .
$$

This implies

$$
w\left(\mathcal{F}^{(2,0)}(T, S, \Omega)(t)\right) \leq \frac{(\lambda t)^{2}}{2(1-k)^{2}} w(\Omega) .
$$

By induction we get

$$
w\left(\mathcal{F}^{(n, 0)}(T, S, \Omega)(t)\right) \leq \frac{(\lambda t)^{n}}{n !(1-k)^{n}} w(\Omega) .
$$

Invoking Theorem 1.1 we obtain

$$
w\left(\mathcal{F}^{(n, 0)}(T, S, \Omega)\right) \leq \frac{(\lambda T)^{n}}{n !(1-k)^{n}} w(\Omega) .
$$


Since $\lim _{n \rightarrow \infty} \frac{(\lambda T)^{n}}{n !(1-k)^{n}}=0$, then there is an $n_{0}$ with $\frac{(\lambda T)^{n_{0}}}{n_{0} !(1-k)^{n_{0}}}<1$. This implies

$$
w\left(\mathcal{F}^{\left(n_{0}, 0\right)}(T, S, \Omega)\right)<w(\Omega)
$$

This gives the proof of the second step.

Step 3: $T$ is sequentially weakly continuous. Let $\left\{x_{n}\right\}$ be a sequence in $C([0, T], X)$ such that $x_{n} \rightarrow x$ for some $x \in C([0, T], X)$. By Theorem 1.2 we have $x_{n}(t) \rightarrow x(t)$ in $X$ for all $t \in[0, T]$. By assumption (i) we have $g\left(s, x_{n}(s)\right) \rightarrow g(s, x(s))$ for all $s \in[0, T]$. The use of the Lebesgue dominated convergence theorem for Pettis integral [23, Corollary 4] gives $\left(T x_{n}\right)(t) \rightarrow(T x)(t)$ for all $t \in[0, T]$. Using again Theorem 1.2 , we obtain $T x_{n} \rightarrow T x$. Thus, $T$ is sequentially weakly continuous.

Applying Theorem 2.1, we get a fixed point for $S+T$ and hence a continuous solution to $(3.1)$.

\section{Final remarks}

It is worth noticing that Theorem 3.1 generalizes the corresponding results in [20] and [13]. In [20] $X$ is assumed to be reflexive and an additional hypothesis $\left(\mathrm{H}_{3}\right)$ is also required, while $f \equiv 0$ in [13].

\section{Competing interests}

The authors declare that they have no competing interests.

\section{Authors' contributions}

All authors contributed equally and significantly in writing this article. All authors read and approved the final manuscript.

\section{Author details}

${ }^{1}$ Department of Mathematics, King Abdulaziz University, P.O. Box 80203, Jeddah, 21589, Saudi Arabia. ${ }^{2}$ Centre Universitaire Polydisciplinaire de Kelaa des Sraghnas, Marrakech, Morocco. ${ }^{3}$ Laboratoire de Mathématiques et de Dynamique de Populations, Université Cadi Ayyad, Marrakech, Morocco.

\section{Acknowledgements}

The authors would like to express their thanks to the editor and referees for their helpful comments and suggestions. This work was funded by the Deanship of Scientific Research (DSR), King Abdulaziz University, Jeddah, under Grant No. (130-053-D1433). The authors, therefore, acknowledge with thanks DSR technical and financial support.

Received: 24 April 2013 Accepted: 5 July 2013 Published: 22 July 2013

\section{References}

1. O'Regan, D: Existence results for nonlinear integral equation. J. Math. Anal. Appl. 192, 705-726 (1995)

2. O'Regan, D: Singular integral equations arising in draining and coating flows. Appl. Math. Comput. 205(1), $438-441$ (2008)

3. De Blasi, FS: On a property of the unit sphere in Banach spaces. Bull. Math. Soc. Sci. Math. Roum. 21, 259-262 (1977)

4. Emanuelle, G: Measures of weak noncompactness and fixed point theorems. Bull. Math. Soc. Sci. Math. Roum. 25, 353-358 (1981)

5. Arino, O, Gautier, S, Penot, JP: A fixed point theorem for sequentially continuous mappings with application to ordinary differential equations. Funkc. Ekvacioj 27(3), 273-279 (1984)

6. Kubiaczyk, I: On a fixed point theorem for weakly sequentially continuous mapping. Discuss. Math., Differ. Incl. 15 15-20 (1995)

7. Garcia-Falset, J: Existence of fixed points and measure of weak noncompactness. Nonlinear Anal. 71, 2625-2633 (2009)

8. Taoudi, MA: Krasnosel'skii type fixed point theorems under weak topology features. Nonlinear Anal. 72, 478-482 (2010)

9. Barroso, CS: Krasnoselskii's fixed point theorem for weakly continuous maps. Nonlinear Anal. 55, 25-31 (2003)

10. Hussain, N, Khan, AR, Agarwal, RP: Krasnosel'skii and Ky Fan type fixed point theorems in ordered Banach spaces. J. Nonlinear Convex Anal. 11(3), 475-489 (2010)

11. Agarwal, RP, O'Regan, D, Taoudi, MA: Browder-Krasnoselskii-type fixed point theorems in Banach spaces. Fixed Point Theory Appl. 2010, Article ID 243716 (2010)

12. Agarwal, RP, O'Regan, D, Taoudi, MA: Fixed point theorems for ws-compact mappings in Banach spaces. Fixed Point Theory Appl. 2010, Article ID 183596 (2010)

13. Agarwal, RP, O'Regan, D, Taoudi, MA: Fixed point theorems for convex-power condensing operators relative to the weak topology and applications to Volterra integral equations. J. Integral Equ. Appl. 24(2), 167-181 (2012) 
14. Agarwal, RP, Nawab, H, Taoudi, MA: Fixed point theorems in ordered Banach spaces and applications to nonlinear integral equations. Abstr. Appl. Anal. 2012, Article ID 245872 (2012)

15. Latrach, K, Taoudi, MA, Zeghal, A: Some fixed point theorems of the Schauder and the Krasnosel'skii type and application to nonlinear transport equations. J. Differ. Equ. 221, 256-271 (2006)

16. Latrach, K, Taoudi, MA: Existence results for a generalized nonlinear Hammerstein equation on $L_{1}$ spaces. Nonlinear Anal. 66, 2325-2333 (2007)

17. Mitchell, AR, Smith, CKL: An existence theorem for weak solutions of differential equations in Banach spaces. In: Lakshmikantham, V (ed.) Nonlinear Equations in Abstract Spaces, pp. 387-404. Academic Press, San Diego (1978)

18. Dobrakov, I: On representation of linear operators on $C_{0}(T, X)$. Czechoslov. Math. J. 21, 13-30 (1971)

19. Smart, DR: Fixed Point Theorems. Cambridge University Press, Cambridge, (1980)

20. Barroso, CS, Teixeira, EV: A topological and geometric approach to fixed points results for sum of operators and applications. Nonlinear Anal. 60, 625-650 (2005)

21. Reinermann, J: Fixpunktsatze vom Krasnoselskii-Typ. Math. Z. 119, 339-344 (1971)

22. Dunford, N, Schwartz, JT: Linear Operators, Part I: General Theory. Interscience, New York (1958)

23. Geitz, RF: Pettis integration. Proc. Am. Math. Soc. 82, 81-86 (1981)

doi:10.1186/1687-1812-2013-196

Cite this article as: Hussain and Taoudi: Krasnosel'skii-type fixed point theorems with applications to Volterra integral equations. Fixed Point Theory and Applications 2013 2013:196.

\section{Submit your manuscript to a SpringerOpen ${ }^{\circ}$ journal and benefit from:}

- Convenient online submission

- Rigorous peer review

- Immediate publication on acceptance

Open access: articles freely available online

- High visibility within the field

- Retaining the copyright to your article 